\title{
A percepção dos profissionais de saúde sobre os cuidados das mães de crianças entre 0 a 6 anos usuárias da Estratégia de Saúde da Família
}

I ${ }^{1}$ Astrid Eggert Boehs, ${ }^{2}$ Edilza Maria Ribeiro, ${ }^{3}$ Márcia Grisotti,

${ }^{4}$ Ana Paula Saccol, ${ }^{5}$ Pamela Camila Fernandes Rumor I

Resumo: O artigo procura identificar e analisar a percepção dos profissionais sobre o cuidado que as mães realizam em relação às crianças de 0 a 6 anos. Foram entrevistados profissionais da Equipe de Saúde da Família: médicos, enfermeiros e técnicos/auxiliares de enfermagem de dois bairros do município de Florianópolis/SC. Após a análise de conteúdo dos dados, identificou-se que, apesar de os profissionais perceberem que as mães continuam utilizando os cuidados do setor popular, eles alegam que o aprendizado dos cuidados básicos da criança na família sofreu mudanças. Esse aspecto, aliado às consequências oriundas da implantação de um novo desenho dos serviços públicos de saúde, especialmente em relação à atenção básica, disponibilizada em grande escala à população, tende a tornar as mães mais dependentes dos serviços de saúde e o cuidado das crianças mais medicalizado.

Palavras-chave: cuidado da criança, cultura, saúde da família.

\author{
1 Doutora em Enfermagem \\ pela Universidade Federal \\ de Santa Catarina (UFSC). \\ Professora do Departamento \\ de Enfermagem e do Programa \\ de Pós-Graduação em \\ Enfermagem da UFSC. Líder do \\ NEPEPS. Endereço eletrônico: \\ astridboehs@hotmail.com \\ 2 Doutora em Enfermagem pela \\ UFSC. Professora Titular do \\ Departamento de Enfermagem \\ da UFSC. Membro do NEPEPS. \\ Endereço eletrônico: edilzamr@ \\ linhalivre.net

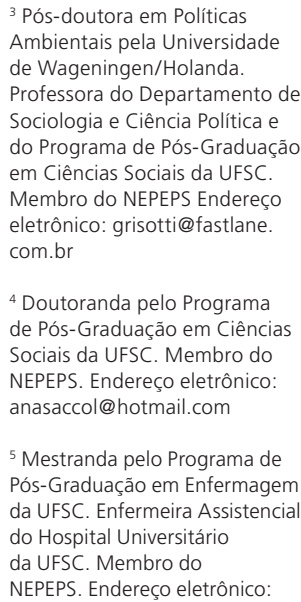

${ }^{4}$ Doutoranda pelo Programa de Pós-Graduação em Ciências Sociais da UFSC. Membro do NEPEPS. Endereço eletrônico: anasaccol@hotmail.com

${ }^{5}$ Mestranda pelo Programa de Pós-Graduação em Enfermagem da UFSC. Enfermeira Assistencial do Hospital Universitário da UFSC. Membro do NEPEPS. Endereço eletrônico: \\ pamrumor@hotmail.com
}


Pesquisas realizadas nas décadas de 1980 e 1990, ocorridas durante o processo de concepção e implantação do Sistema Único de Saúde (SUS) no Brasil, apontavam para a multiplicidade de usos de recursos terapêuticos alternativos ou populares, e a recorrência às distintas fontes de tratamento por parte da população de baixa renda. As explicaçôes para esse fenômeno oscilavam entre a falta de recursos médicos oficiais dessas comunidades e o caráter simbólico das práticas médicas não oficiais, acessíveis e reconhecidas pela população. No caso brasileiro, alguns estudos revelaram que isso não significava uma rejeição da medicina oficial, mas uma estratégia de complementaridade no processo de tratamento (LOYOLA, 1984; 1987; BUCHILLET, 1991; GRISOTTI, 1992).

Após quase duas décadas desde a publicação dessas, percebemos a necessidade da revisão desses estudos em função das consequências oriundas da implantação de um novo desenho dos serviços públicos de saúde, especialmente em relação à atenção básica, disponibilizados em grande escala à população. $\mathrm{O}$ Ministério da Saúde lançou, em 1994, o Programa de Saúde da Família (PSF), que foi sendo consolidado ao longo dos anos, expandindo-se para estratégia prioritária de reorganização da atenção básica no Brasil, passando a ser denominado de Estratégia de Saúde da Família (ESF) (BRASIL, 2006). A atenção básica considera o sujeito em sua singularidade, na complexidade e na inserção sociocultural, e busca a promoção da saúde, a prevenção, o tratamento das doenças e a redução de danos que possam comprometer a vida saudável. A ESF possui, entre seus princípios, atuar no território realizando diagnóstico situacional, buscando cuidado do indivíduo e das famílias ao longo do tempo (BRASIL, 2006). A inserção da ESF em 5.261 dos municípios brasileiros (94,53\%), com a atuação de 30.603 equipes, bem como uma cobertura populacional de 51,0\% (BRASIL, 2010), representa fatores decisivos na ampliação do acesso da população aos recursos oficiais de saúde, destacando-se, para os fins deste artigo, a clientela que acorre aos programas desenvolvidos na ESF, em especial as mães de crianças de 0 a 6 anos.

Recentemente, alguns estudos buscaram conhecer como a equipe de saúde reage diante do uso de práticas de cuidado oriundas do sistema familiar de cuidado, também denominado de "informal" ou "popular" (BARBOSA et al., 2004; ELSEN; MONTICELLI, 2006; SCHOLZE; SILVA, 2005), mais especificamente com relação ao cuidado da criança. Buscaram, ainda, analisar a influência das 
crendices e práticas populares na assistência de enfermagem segundo os enfermeiros de um PSF de um município do nordeste brasileiro (MOREIRA et al., 2006).

Com base nas discussões propostas por tais estudos, o objetivo da presente pesquisa $^{1}$ foi identificar as percepções dos profissionais das equipes da ESF sobre os cuidados que as mães dispensam às crianças de 0 a 6 anos, no que se refere ao uso de recursos provenientes do saber popular, familiar e profissional.

\section{Métodos}

Foi realizado um estudo qualitativo. Os dados foram coletados em duas Unidades Básicas de Saúde (UBS) situadas em dois bairros do município de Florianópolis, Santa Catarina: um na região leste (que aqui chamaremos de bairro $A$ ) e um na região norte (bairro B). A população é constituída de nativos e migrantes do interior do estado e de outros estados brasileiros, essencialmente de baixa renda socioeconômica. O bairro A está localizado nas proximidades do centro de Florianópolis, possuindo, além da ESF, o programa municipal denominado Capital Criança, que é focado na assistência da gestante e puérpera e na criança de 0 a 10 anos; além disso, mantém uma relação estreita com os cursos da área da saúde da Universidade Federal de Santa Catarina (UFSC) formalizado na Rede Docente Assistencial (RDA). O bairro B, distante do centro da cidade, também possui a ESF e o Capital Criança.

Os sujeitos do estudo foram médicos, enfermeiros e técnicos ou auxiliares de enfermagem integrantes das diferentes áreas da ESF nos dois bairros. No total, realizaram-se 18 entrevistas: 12 no bairro $A-4$ enfermeiros, 4 médicos (uma pediatra) e 4 técnicos/auxiliares de enfermagem - e 6 profissionais no bairro $B$ um enfermeiro, 2 médicos e 3 técnicos/auxiliares de enfermagem.

As entrevistas foram agendadas previamente mediante contato telefônico, realizadas individualmente a partir de um roteiro de perguntas semiestruturado, e, em seguida, gravadas e transcritas. Para a análise dos dados, foram seguidas as etapas da análise de conteúdo: organização dos dados, codificação, categorização, inferências e interpretação. A categorização inicial correspondeu aos itens previamente estabelecidos no formulário de entrevista. Adotaram-se princípios como "exaustividade", "representatividade", "homogeneidade" e "pertinência" (BARDIN, 2002). A interpretação dos dados foi efetuada à luz de autores com base no referencial dos subsistemas de cuidado - popular, familiar e profissional - proposto por Kleinman (1980; 1990). 
O projeto de pesquisa foi aprovado pelo Comitê de Ética em Pesquisas com Seres Humanos da UFSC, em abril de 2006, sob o número 022/06. Realizouse de acordo com a Resolução no 196/96 do Conselho Nacional de Saúde (BRASIL, 1996) para pesquisas com seres humanos, no que diz respeito ao sigilo, anonimato, consentimento livre e esclarecido obtido junto aos profissionais antes da entrevista, concordância em gravar os dados e liberdade de desistência em qualquer momento da pesquisa.

\section{Resultados}

\section{Características dos profissionais}

No que tange aos médicos do Bairro A, três eram do sexo feminino e um do sexo masculino. Do bairro $\mathrm{B}$, os dois médicos entrevistados eram do sexo feminino. Todos tinham idade superior a 25 anos e atuavam na área da atenção básica, sendo que apenas uma médica do bairro A atuava, concomitantemente, na área hospitalar. Os profissionais médicos do bairro A tinham uma ou mais especializações, tais como saúde da família/medicina de família e comunidade, saúde pública, homeopatia, nutrologia e pediatria. Uma médica possui mestrado. No bairro B, uma era especialista em pediatria e em saúde pública, e a outra, especialista em homeopatia e acupuntura. No que se refere ao tempo de serviço na UBS, um médico do bairro A tinha entre um e 5 anos de serviço. Outro, entre 5 e 10 anos e dois, acima de 10 anos. No bairro B, uma das médicas tinha tempo inferior a um ano, e a outra, entre 5 e 10 anos de serviço na UBS.

Quanto ao perfil dos enfermeiros, dos quatro entrevistados no bairro A, três eram do sexo feminino e um do sexo masculino. No bairro B, apenas uma enfermeira foi entrevistada. Todos os enfermeiros tinham idade superior a 25 anos e atuavam somente na área da atenção básica. Três enfermeiras do bairro A tinham especialização em gestão de saúde pública. A enfermeira do bairro $\mathrm{B}$ era especialista em administração hospitalar. Dois dos enfermeiros do bairro A tinham entre um e 5 anos de tempo de serviço na UBS, e os outros dois, tempo inferior a um ano. A enfermeira do bairro B tinha entre 5 e 10 anos de tempo de serviço na UBS. Observou-se uma diferenciação, em termos de complementação da formação, entre médicos e enfermeiros: os primeiros eram mais voltados para o atendimento direto à clientela e os enfermeiros, para a área de gestão/gerência. 
Os profissionais de enfermagem de nível médio entrevistados no bairro A e

B caracterizaram-se por serem do sexo feminino e possuírem mais de 25 anos de idade, tendo cursado o ensino médio completo, exceto uma do bairro A, com superior incompleto. Das profissionais do bairro $\mathrm{A}$, uma trabalhava há menos de um ano; duas, entre um e 5 anos; a outra, entre 5 e 10 anos. No bairro B, duas trabalhavam entre um e 5 anos, e outra, entre 5 e 10.

\section{Percepção dos profissionais sobre os cuidados básicos de saúde das mães às crianças de 0 a 6 anos}

Emergiram cinco categorias como resultado da análise: (1) as mães permanecem utilizando cuidados do setor popular; (2) as mães aprendem menos sobre cuidados básicos da criança no sistema familiar; (3) as mães tendem a estar mais dependentes dos serviços de saúde e o cuidado das crianças está mais medicalizado; (4) as mães adquiriram uma nova cultura, a da dipirona e do paracetamol, (5) As condiçōes de vida das famílias não favorecem o cuidado das crianças.

\section{1) As mães permanecem utilizando cuidados do setor popular}

Os profissionais relataram que, no ambiente dos serviços de saúde, as mães falam pouco sobre a busca de cuidados do setor popular, mesmo quando questionadas a respeito do assunto; somente falam se existe um ambiente de "muita confiança" nos profissionais. Reconhecem, no entanto, que em uma abordagem de rotina, eles não priorizam a busca dessas informaçôes, embora, ao mesmo tempo, todos os profissionais entrevistados reconheçam que as mães procuram alternativas para o cuidado da saúde das crianças, entre as quais benzedeiras, curandeiros, homeopatia e outras práticas, e que isso não tem implicado na diminuição da procura do serviço de saúde. Uma médica do bairro A referiu que, para as crises de bronquite, as mães buscam o médico, mas nas bronquites prolongadas, acabam recorrendo à benzedeira ou a outros tipos de curandeiros do setor popular, visto que a medicina oficial ainda não tem solução definitiva para tal problema. Tanto médicos, enfermeiros, como técnicos e auxiliares salientaram que as famílias levam os adultos para práticas populares como candomblé, passes, entre outros, e, quando se trata da criança, buscam mais o médico e medicamentos da farmácia. 
Nos dois bairros há uma grande proliferação das igrejas evangélicas pentecostais, que se instalam muito próximas das casas de famílias com baixa renda. Assim, progressivamente, os profissionais vêm se defrontado com a influência do papel do pastor da igreja no cuidado da saúde das crianças. Médicos e enfermeiras consideram que, de certa forma, isso é positivo, porque as oraçôes representam um reforço quando utilizado de forma concomitante com medicamentos.

Médicos e enfermeiras aceitam as práticas utilizadas pelas mães quando consideram que são seguras para a saúde da criança, e também quando seguem, conjuntamente, as prescrições médicas ou a orientação dos cuidados feitos pelas enfermeiras. Ou seja, essa aceitação é relativa, pois requer uma avaliação prévia, como afirma uma enfermeira: "Pode usar se for supervisionado por um profissional de saúde e realmente não tiver risco" (Enf. 2/ bairro A), ou, como enfatiza um médico: Quando chega uma mãe com uma prática nova é complicado, tem que ter jogo de cintura, não adianta a gente se opor... o melhor é aceitar e estudar, ver se faz mal, voltar a conversar com a pessoa [.] (Med. 1/bairro A).

Entre as razões apontadas para conciliar as práticas populares com a prática dos profissionais, está a necessidade de manter o vínculo já estabelecido ou de reforçá-lo. Além disso, alguns médicos valorizaram a capacidade da família de cuidar no domicílio, mesmo que adotando "manias", "crenças" e "tabus", já que isso traduz autonomia no cuidado da criança e diminui a dependência do profissional de saúde. $\mathrm{Na}$ percepção dos profissionais, os cuidados provenientes do sistema popular e familiar são importantes, pois, mesmo utilizando a consulta médica, em casos de doença, os cuidados em casa fazem com que mães sintam que estão fazendo algo mais do que o remédio, "ajudam a ter paciência para esperar que a doença melhore".

Em consonância com a postura adotada frente aos clientes que buscam alternativas de cuidado, os profissionais enfermeiros e médicos referiram acreditar em outras práticas alternativas no cuidado, mas que utilizam, eles próprios, em primeiro lugar, a alopatia.

As técnicas e auxiliares de enfermagem dos dois bairros demonstraram que possuíam um conhecimento mais amplo sobre as crenças em práticas populares do que os demais profissionais, porém estavam preocupadas com os efeitos negativos de tais práticas para a saúde das crianças: 
O que a benzedeira dá para passar no machucadinho, aí é que mora o perigo, né?[.]

Ainda existem casos de botar moeda no umbigo. Ainda existem casos de a mãe colocar pó de café no umbigo, acredita? [.] Vão passando de mãe pra filha, e elas acreditam... Mas como que eu não vou enfaixar? Isso já vem da avó, da bisavó, como é que isso vai ser tirado assim? (Téc.de Enf 3/bairro A).

Essas profissionais apontaram para a necessidade de erradicar crenças e práticas populares "prejudiciais", como reflete o depoimento a seguir: "A gente explica bastante, passando confiança pra ela poder acreditar, pra que não volte a acontecer" [.] (Aux. de Enf. 2/bairro B).

\section{2) As mães aprendem menos sobre cuidados básicos da criança no sistema familiar}

Para os profissionais de saúde, o processo de aprendizagem dos cuidados no sistema familiar mudou. O vínculo familiar favorecia a manutenção de uma rede de saberes e práticas a qual incluía crenças, tabus, rituais nem sempre considerados corretos pelos profissionais. Esse conhecimento, segundo eles, e a experiência familiar de aprendizagem cultural do cuidado que perpassava as geraçôes, foram se reduzindo, tornando as mães inseguras em relação aos cuidados dos filhos.

O fato de haver muitas famílias migrantes, em ambos os bairros, que se separaram de suas comunidades e de sua parentela, contribuiu, na visão dos profissionais, para a interrupção da transmissão dos cuidados do sistema familiar e popular, visto que estes, em sua grande maioria, existem e continuam através da oralidade e da convivência. Os profissionais relatam que, cada vez mais, as mães chegam à UBS sem ter realizado procedimentos básicos, o que, de acordo com eles, demonstra o despreparo e menor autonomia nos cuidados à saúde de suas crianças.

Ainda dentro dessa perspectiva, os profissionais alegam que a população nativa do município está perdendo costumes tradicionais, como, por exemplo, no caso da alimentação, o pirão de peixe, o pirão de feijão, o feijão e o ovo, enquanto desenvolveram outros, como a sopa de bolacha, cujo excesso é prejudicial para a saúde da criança. Também as mães, atualmente, não conhecem mais os cuidados relacionados com o "quente e frio", a aplicação de banhos e a alimentação em situações específicas como diarréia, anemia e outros. 


\section{3) As mães tendem a estar mais dependentes dos serviços de saúde e o cuidado das crianças está mais medicalizado}

$\mathrm{Na}$ visão das três categorias profissionais, a facilitação do acesso da população aos serviços de saúde é uma realidade nos dois bairros estudados. A declaração de uma técnica de enfermagem é ilustrativa de condições motivadoras da procura dos serviços de saúde:

Eu acho que o serviço de saúde público é bom, a qualidade é boa. Somos o único país em que se ganha anticoncepcional de graça, camisinha de graça, e um monte de medicação de graça (Tec. de Enf. 2/bairro B).

A presença das agentes comunitárias de saúde, adentrando nas casas, na visão dos profissionais entrevistados, também tem facilitado o acesso aos serviços de saúde, uma vez que as mães pedem para as agentes intermediarem a busca de consultas principalmente de urgência. Ademais, auxiliam a desmistificar tabus e crenças populares, colocando as famílias mais perto dos conhecimentos do setor profissional. Ou seja, a consequência mais flagrante, na visão dos profissionais, da facilitação do acesso aos serviços de saúde tem sido a crescente adoção de cuidados "apreendidos" nos serviços de saúde e a medicalização do cuidado. Conforme uma médica entrevistada no bairro $\mathrm{B}$, como as mães não conhecem mais aqueles cuidados ensinados pela rede familiar, tudo precisa ser medicalizado por um profissional de saúde. Outro depoimento ajuda a reforçar a visão de aumento da dependência das pessoas de cuidados médicos: "Eles procuram muito o posto para qualquer coisa, pode ser um cortezinho de nada, fazem questão de mostrar para o profissional. (Téc de Enf 3/bairro A)".

Em caso da unidade de saúde "não dar conta" dos problemas das crianças, as mães também buscam, e geralmente conseguem, o atendimento especializado e hospitalar, como demonstrado no seguinte depoimento:

A gente tem essa facilidade de ter um PA (Pronto Atendimento) aqui perto e que tem pediatra 24 horas, então, muitas vezes eles vão lá em função de ter pediatra, e não em função que seria o atendimento (Méd. 1/bairro B).

Já as médicas de ambos os bairros consideram que a mídia tem um papel importante entre as causas do aumento da dependência da clientela em relação aos serviços de saúde e medicalização. Os anúncios na televisão, nos quais sistematicamente há a recomendação, "procure seu médico", segundo elas, surtiu efeito. Todavia, ponderam que isto é bastante ambíguo, já que tem-se um lado 
positivo, o das pessoas de prevenirem a automedicação, o que facilita as intervenções

precoces perante problemas complexos; mas, por outro lado, incentiva a dependência de especialistas e o desuso de práticas de cuidado úteis, anteriormente adotadas pelas mães, em casos de doenças e sintomas comuns evitáveis.

\section{4) As mães adquiriram uma nova cultura, a da dipirona e do paracetamol}

A “cultura da dipirona e do paracetamol”, referida pelos profissionais, pode ser traduzida como o atendimento imediatista de cuidados em saúde. No depoimento de uma enfermeira:

As mães adotaram a cultura do paracetamol. Não sei até que ponto isto é uma coisa positiva ou não. $\mathrm{O}$ paracetamol não é tão inofensivo assim pra ser usado, e a gente também não pode ficar fomentando aqui o uso de outros tipos de medicamentos sem o acompanhamento da equipe de saúde (Enf. 4/ bairro A).

As auxiliares de enfermagem, principalmente do bairro B, mostram suas experiências com respeito à criança com febre, um dos maiores motivos da busca pela Unidade Básica de Saúde. Explicam que, diante da febre, as mães procuram a UBS já tendo usado dipirona ou o paracetamol que possuem em casa.

A dependência das mães do sistema profissional de saúde tem propiciado a incorporação do uso de medicamentos como forma de resolução de desconfortos e pequenas alterações no estado de saúde das crianças, antes atendidas com medidas do sistema familiar e popular, tais como uso de calor/frio, banhos e chás. $\mathrm{O}$ depoimento abaixo se refere à situação mencionada: "60\% das mães são pessoas que já conhecem, têm em casa, analgésicos, antitérmicos e dão para a criança quando elas têm algum problema, uma dor, uma febre” (Med 2/bairro A).

Os profissionais afirmam que as mães tendem a não compreender que há um tempo de espera para o tratamento fazer efeito e o corpo se recuperar. De acordo com eles, as mães querem a solução rápida: se estão tomando antibiótico, "querem estar recuperados no dia seguinte". O depoimento de uma medica retrata a situação:

As mães hoje não têm paciência. Se você não quer ver a pessoa todo dia aqui no posto, tem que explicar muito bem o que pode acontecer. Se souber que febre vai continuar nas próximas 48 horas, a mãe então espera por isso. Tudo está mais rápido, o computador está mais rápido, mas o tempo biológico não mudou. A cura da gripe tem seu tempo, assim como da diarréia (Med 4/ bairro A). 
Ainda na visão dos profissionais, certas mães, quando não veem os problemas de saúde das crianças resolvidos de imediato, buscam novos atendimentos em outros serviços e junto a outros médicos, gerando, para um mesmo problema, duas ou três abordagens diferentes. Quanto mais abordagens, mais inseguras se tornam as mães. Estas também tendem a rejeitar o médico clínico geral para o atendimento da criança, uma vez que a expectativa é de um pediatra, o que contraria a lógica da ESF. Essa rejeição, segundo os profissionais, é uma das razões que leva as mães a buscarem as emergências pediátricas, porque lá elas têm certeza de que irão encontrar tal profissional.

\section{5) As condiçôes de vida das famílias}

\section{não favorecem o cuidado das crianças}

Finalmente, os profissionais de ambos os bairros, ainda reconhecem que o processo saúde-doença das famílias é dependente das várias condições e circunstâncias que influem na qualidade e na execução do cuidado prestado às crianças pelas mães. Destacam as condições sócio-econômicas em primeiro lugar, seguidas da baixa escolaridade e da sua pouca idade. As mães precisam trabalhar logo depois do parto, por questóes econômicas, prejudicando assim o período de amamentação, que, inclusive, faz parte de um programa federal de incentivo à qualidade de vida da criança. Soma-se a isso o fato de as avós das crianças e outras pessoas da rede familiar também trabalharem fora ou morarem distantes, contribuindo para a diminuição da rede de apoio no cuidado à criança.

O depoimento de uma enfermeira ilustra essa situação e mostra a impotência e os limites enfrentados pelo profissional nas suas recomendações terapêuticas quando confrontados com as condições de vida das mães: "No bairro, as mães desmamam precocemente pela pressa de voltarem a trabalhar, porque não tem ninguém que ajude. Não adianta insistir. (Enf 2/bairro A)”

\section{Discussão}

O perfil dos profissionais entrevistados aponta para a predominância do gênero feminino mesmo entre os profissionais médicos. Entre estes e os enfermeiros há uma busca para estudos de pós-graduação; inclusive, há duas médicas que fizeram cursos ligados à homeopatia e acupuntura. Os profissionais da enfermagem atuam somente na atenção básica, e entre os profissionais médicos apenas um 
também atua na área hospitalar, indicando que esses profissionais concentram seu trabalho nesse nível de atenção.

O perfil dos profissionais de nível superior possibilitou a percepção de que as famílias utilizam os recursos do setor familiar e popular, e mostraram a importância dessa utilização para a produção da saúde nas famílias, diferentemente dos auxiliares de enfermagem que entendem ser necessária a erradicação de práticas que não sejam aquelas do setor profissional. No entanto, mesmo trabalhando na atenção básica com a ESF, cujo foco está na família e na promoção da saúde, reconhecem que, em uma abordagem de rotina, não buscam informações sobre a utilização desses recursos. Referem também que as mães tendem a não revelar a utilização de outras terapias, principalmente a busca de benzedeiras. Esse resultado está de acordo com o estudo de Gentil, Robles e Grossemann (2010) com mães de crianças em um Hospital de Florianópolis, SC, no qual houve a prevalência do uso de terapias complementares de $87,6 \%$ por parte das mães. A utilização dessas terapias só foi revelada aos médicos por 57,6\% delas, sendo que as orações, tratamentos espíritas e benzeduras foram menos revelados do que o uso de xaropes, massagens, homeopatia, reiki e florais.

A vigência de novas condições no viver das famílias favorecendo a diminuição do processo de aprendizagem dentro do setor familiar e popular, conforme apontaram os profissionais, encontra respaldo na literatura (CARVALHO; ALMEIDA, 2003; SARTI, 2004). As novas condições advêm, entre outros fatores, da inserção feminina no mercado de trabalho, reduzindo o tempo da mulher para o cuidado dos filhos; da diminuição da influência das avós na socialização da família; da perda das referências culturais em função da maciça migração da população rural para cidades de grande e médio portes; da fragilidade e insegurança provenientes da precária infraestrutura destinada às comunidades de baixa renda.

Para Simionato e Oliveira (2003, p. 62), a família também tem se transformado, no sentido de se tornar mais influenciável do que influente e que:

Com o movimento higienista do século XIX, que ela começou a perder a sua capacidade de cuidar da saúde e educação de seus membros, tornando-se bastante dependente dos profissionais da saúde e da educação. (SIMIONATO e OLIVEIRA, 2003, p. 62)

Tesser (2006a, p. 66), referindo-se aos efeitos da perda dos saberes familiar e popular, afirma que: “[...] o conhecimento cultural sobre a saúde (tradicional, popular ou de outras medicinas), de gerações anteriores e do entorno social do 
doente (fonte de tratamento integrado à cultura e à vida do paciente e dos seus), transformam-se em virtudes obsoletas e muitas vezes indesejadas".

Já autores da sociologia contemporânea, entre eles Giddens (1991, p. 35), têm destacado, em suas análises sobre os mecanismos de desencaixe envolvidos no desenvolvimento das instituições modernas, a dependência cada vez maior dos indivíduos em relação aos conhecimentos especializados ou peritos, mesmo conhecendo pouco sobre eles. O autor define os sistemas peritos como "sistemas de excelência técnica ou competência profissional que organizam grandes áreas dos ambientes material e social em que vivemos hoje". De acordo com Giddens (1991, p. 35-36), embora apenas em certas ocasióes a maioria das pessoas leigas consulte "profissionais", como advogados, médicos, arquitetos, etc., os sistemas em que os conhecimentos especializados estão inseridos influenciam regularmente muitos aspectos da vida cotidiana dos indivíduos.

Ao estar simplesmente em casa, estou envolvido num sistema perito, ou numa série de tais sistemas, nos quais deposito minha confiança. Conheço muito pouco os códigos de conhecimento usados pelo arquiteto e pelo construtor no projeto e construção da casa, mas não obstante tenho "fé" no que eles fizeram. Minha "fé" não é tanto neles, embora eu tenha que confiar em sua competência, como na autenticidade do conhecimento perito (expert knowledge) que eles aplicam algo que não posso, em geral, conferir exaustivamente por mim mesmo. [...] (GIDDENS, 1991, p. 35-36).

Assim, tendo ampliado o contato em função de sucessivas vindas das mães à Unidade Básica de Saúde, e frente à diminuição de conhecimentos e experiência para o cuidado cotidiano de doenças e sintomas comuns evitáveis, anteriormente advindos do sistema familiar e popular, as mães passam a recorrer mais amplamente aos especialistas profissionais. Estabeleceu-se uma via de mão dupla: de um lado, as mães com menos conhecimentos e habilidades no cuidado em saúde de seus filhos procuram mais os serviços de saúde, a cujo acesso foi facilitado. De outro, trabalhadores da saúde, imersos no "saber" e "modus operandi" do sistema profissional, influenciam as famílias a se comportarem conforme o modelo médico, realimentando influências/dependência, o que contribui para ampliar a demanda e aumentar os custos do atendimento em saúde.

Apesar dos princípios de promoção da saúde serem preconizados pela ESF e seu enfoque na família, a prática cotidiana dos profissionais das Unidades Básicas de Saúde ainda se pauta, predominantemente, pelo modelo biomédico, conforme é reportado e caracterizado por diversos autores (TESSER, 2006a; 2006b; FRANCO, MERHY, 2003; RIBEIRO, 2005; CONNIL, 2008; SOUSA, 2008). 
A medicalização de inúmeros tipos de problemas, queixas, dores, incômodos, tendo como exemplo gripes, resfriados, tristezas, lutos, pequenas contusões, nascimento, morte, pânico, tem gerado uma crescente e infindável demanda de atendimento médico, seja no cotidiano dos serviços do SUS, seja na rede básica ou nas ESF (TESSER, 2006a; 2006b). É o que percebem os entrevistados desse estudo na sua prática: há o declínio da autonomia das mães com dependência cada vez maior dos profissionais e dos medicamentos alopáticos.

A contraprodutividade dos serviços biomédicos do SUS se evidenciaria pela produção de efeitos paradoxais decorrentes excesso de produção da ferramenta e/ou monopolização da função, deixando de operar dentro de seus objetivos e de produzir corresponsabilidade e autonomia da população (TESSER, 2006b). Podemos, assim, perceber a contradição ainda existente na atenção básica, no que diz respeito à promoção da saúde e à utilização de recursos de baixo custo para sua prevenção e reabilitação, bem como apontar a força do lobby mercadológico das grandes indústrias farmacêuticas no setor saúde.

\section{Conclusões}

Retornando ao objetivo, que foi identificar a percepção dos profissionais das equipes da ESF sobre o uso de recursos de cuidado do setor popular, familiar e profissional por mães de crianças de 0 a 6 anos, cumpre enfatizar o maior achado deste estudo, que foi a percepção dos profissionais em destacar que há uma relação entre a diminuição da autonomia das mães no cuidado a saúde de seus filhos e o desenvolvimento de uma progressiva medicalização dos cuidados das crianças pela maior oferta dos serviços com a implantação da ESF. Semelhante percepção dos profissionais constitui, portanto, um parodoxo, já que os princípios da ESF buscam justamente o contrário: a mudança do modelo de atenção visando favorecer a assistência no nível da atenção básica de forma longitudinal, tendo como foco principal a família e a comunidade na qual ela está inserida. Assim, novas perguntas de pesquisa de cunho avaliativo deverão ser delineadas após quase duas décadas de implantação do Programa de Saúde da Família. Questiona-se se, apesar dos números quantitativos positivos tão propalados sobre o aumento da cobertura dos municípios e da população atendida pela ESF, ainda não amadurecemos qualitativamente para focar e trabalhar com a família. Questiona-se também o que os profissionais entendem sobre o conceito 
de família e de saúde da família, e em que medida estes são apenas discursos e conceitos abstratos para os profissionais, e mesmo para as instituições que formam os novos profissionais.

Dadas as limitações desta pesquisa, realizada com um pequeno número de profissionais em apenas dois locais de um município no sul do país, mais pesquisas precisam elucidar a abordagem dos profissionais na ESF, bem como em que medida estes estão incorporando um novo modelo de atenção ou se continuam se pautando apenas no modelo estritamente biológico. Mais especificamente: como os profissionais de saúde estão trabalhando com a família da criança? Que dados são levantados?

Com relação aos profissionais de enfermagem de nível médio, novos estudos devem ser feitos para iluminar melhor como estão manejando as práticas de saúde do setor popular e familiar, visto que os dados desta pesquisa mostraram que esses profissionais valorizam sobremaneira as práticas do modelo biológico.

Nas transformações indicadas para o sistema de saúde, é importante que se leve em conta a necessidade de avanços, de modo a ter-se o melhor equilíbrio entre o poder do setor profissional e a saudável valorização da autonomia das famílias. Deve-se levar igualmente em conta a importância de estabelecer maior diálogo entre as partes, no sentido de compartilhar, e não impor, conhecimentos e experiências, visando seguir as premissas da atenção básica e os princípios da ESF.

\section{Referências}

BARBOSA, M.A. et al. Crenças populares e recursos alternativos como práticas de saúde. Rev. Enferm UERJ, v. 12, n. 1, p. 38-43, 2004.

BARDIN, L. Análise de Conteúdo. Lisboa: Ediçôes 70, 2002.

BRASIL. Conselho Nacional de Saúde. Comissão Nacional de Ética em Pesquisa. Resolução $n^{o} 196$ de 10 de outubro de 1996: diretrizes e normas regulamentadoras de pesquisa envolvendo seres humanos. Brasília: Ministério da Saúde, 1996.

BRASIL. Ministério da Saúde. Departamento de Atenção Básica. Números da Saúde da Família. Disponível em: http://200.214.130.35/dab/abnumeros.php\#numeros Acesso em: 27 set 2010 .

. Portaria no 648/GM de 28 de março de 2006. Aprova a Política Nacional de

Atenção Básica. Disponível em: http://www.saude.sc.gov.br/gestores/Pacto_de_Gestao/ portarias/GM-648.html Acesso em: 01 out 2010. 
BUCHILlET, D. (Org.) Medicinas Tradicionais e Medicina Ocidental na Amazônia. Belém (PA): CEJUD, 1991.

CARVALHO, I.M.M.; ALMEIDA, P.H. Família e proteção social. São Paulo Perspec., v. 17, n. 2, p. 109-22, 2003.

CONNIL, E.M. Ensaio histórico-conceitual sobre a Atenção Básica de Saúde: desafios para a organização de serviços e da Estratégia de saúde da Família em centros urbanos. Cad. Saúde Publica, v. 24, supl 1, p. 7-16, 2008.

ELSEN, I.; MONTICELLI, M. A cultura como obstáculo: percepções da enfermagem no cuidado às famílias em alojamento conjunto. Texto Contexto Enferm., v. 15, n. 1, p. 26-34, 2006. FRANCO, T.B.; MERHY, E.E. Programa de Saúde da Família (PSF): contradições de um programa destinado a mudança do modelo tecnoassistencial. In: MERHY, E.E. (Org.). O trabalho em saúde: olhando e experienciando o SUS no cotidiano. São Paulo: Hucitec, 2003. GENTIL,L.B.; ROBLES,A.C.; GROSSEMANN, S. Uso de terapias complementares por mães em seus filhos: estudo em um hospital universitário. Ciênc Saúde Coletiva, v.15, supl.1, p. 1293-1299 junho de 2010

GIDDENS, A. As conseqüências da Modernidade. São Paulo: UNESP, 1991.

GRISOTTI, M. Sistemas médicos oficial, familiar e paralelo: estudo de percepção e comportamento em relação ao processo saúde-doença em comunidades de baixa renda. 1992. Dissertação (Mestrado em Ciências Sociais) - Universidade Federal de Santa Catarina, Florianópolis, 1992.

KLEINMAN, A.; CSORDAS, T.J. The therapeutic process. In: JOHNSON, T.M.; SARGENT, C.F. (Org.). Medical anthropology: contemporary theory and method. Westport: Greenwood Press, 1990. p. 11-25.

KLEINMAN, A. Patients and healers in the context of the culture: an exploration of the borderland between anthropology, medicine and psychiatry. Califórnia: Regents, 1980.

LOYOLA, M.A. Médicos e curandeiros: conflito social e saúde. São Paulo: Difel, 1984. Medicina Popular: rezas e curas de corpo e alma. Ciência Hoje, v. 6, n. 35, p. 34-43, 1987.

MOREIRA, C.T. et al.. Crendices e práticas populares: influência na assistência de enfermagem prestada à criança no programa saúde da família. RBPS, v. 16, n.1, p. 11-8, 2006.

RIBEIRO, E.M. Concreticidade do vínculo do/no Programa de Saúde da Família (PSF): desafios de médicos e enfermeiras em uma realidade de implantação do Programa. 2005.Tese (Doutorado em Enfermagem) - Programa de Pós-graduação em Enfermagem, Universidade Federal de Santa Catarina, Florianópolis, 2005.

SARTI, C.A. A família como ordem simbólica. Psicol. USP, v. 15, n.3, p. 11-28, 2004. 
SCHOLZE, A.S.; SILVA, Y.F. Riscos potenciais à saúde em itinerários de cura e cuidado. Cogitare Enferm., v.10, n.2, p. 9-16, 2005.

SIMIONATO, M.A.W.; OLIVEIRA, R.G.O. Funções e transformações da família ao longo da história. In: Encontro Paranaense de Psicopedagogia, 1, 2003. I Encontro Paranaense de Psicopedagogia, Anais... CD ROM, ABPppr., nov., 2003.

SOUSA, F.G.M. Tecendo a teia do cuidado à criança na atenção básica de saúde: dos seus contornos ao encontro com a integralidade. 2008. Tese (Doutorado em Enfermagem) Programa de Pós-graduação em Enfermagem, Universidade Federal de Santa Catarina, Florianópolis, 2008.

TESSER, C.D. Medicalização Social (I): o excessivo sucesso do epistemicídio moderno na saúde. Interface, v.9, n.18, p.61-76, 2006a.

. Medicalização Social (II): limites biomédicos e propostas para a clínica na atenção básica. Interface, v.10, n.20, p.347-62, 2006b.

\section{Nota}

${ }^{1}$ Projeto de pesquisa financiado pelo CNPq, Processo 401914/2005-4. Desenvolvido junto ao Núcleo de Extensão e Pesquisa em Enfermagem e Promoção da Saúde - NEPEPS do Programa de PósGraduação em Enfermagem da UFSC no período de 2006 a 2008. Com apoio da Secretaria de Saúde da Prefeitura Municipal de Florianópolis. 


\section{Abstract}

Health professionals perception about mothers caring for children between 0 to 6 years old who are users of the Family Health Strategy

This paper seeks to identify and analyze the professionals' perception about mothers who care for children between 0 and 6 years old. Professionals from the Family Health Strategy were interviewed: doctors, nurses and nursing technicians/assistants. The research took place in two districts of Florianópolis/SC. Through content analysis, we identified that, although professionals realize that mothers keep on using public health care, they claim that learning child's basic care in the family subsystem has changed, as familiy life conditions do not encourage home care. This aspect, along with the consequences of implementation of a new design of public health services particularly for basic care and available on a large scale to the population, tend to make women more dependent on health services and children's care more medicalized.

Key words: child care, culture, family health. 